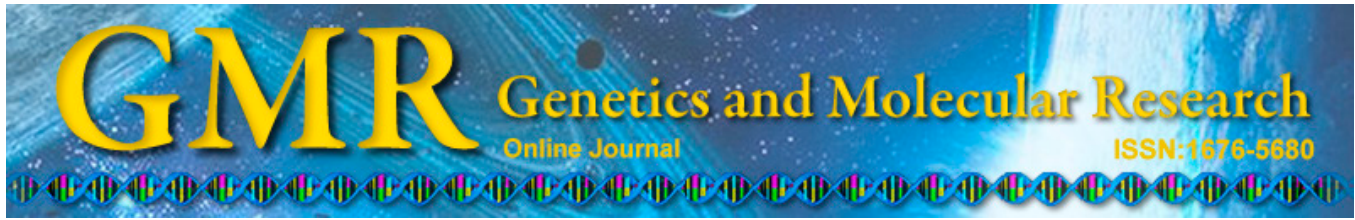

\title{
Association of a miR-34b binding site single nucleotide polymorphism in the 3'-untranslated region of the methylenetetrahydrofolate reductase gene with susceptibility to male infertility
}

\author{
W. Zhang ${ }^{1}$, W.-Q. Lin ${ }^{1}$, H.-F. Cao ${ }^{1}$, C.-Y. Li ${ }^{1}$ and F. Li ${ }^{2}$ \\ ${ }^{1}$ The Reproductive Medicine Center, \\ First Affiliated Hospital of Zhejiang University, Hangzhou, China \\ ${ }^{2}$ The Reproductive Medicine Center, \\ Fourth Affiliated Hospital of Jiangsu University, Zhenjiang, China \\ Corresponding author: F. Li \\ E-mail: lifeng201403@163.com
}

Genet. Mol. Res. 14 (4): 12196-12204 (2015)

Received March 30, 2015

Accepted June 24, 2015

Published October 9, 2015

DOI http://dx.doi.org/10.4238/2015.October.9.8

\begin{abstract}
This study aims to explore the possible associations between a genetic variation in the miR-34b binding site in the 3'-untranslated region (UTR) of the methylenetetrahydrofolate reductase (MTHFR) gene (rs55763075) with male infertility in a Chinese population. Genotype distributions of the rs55763075 single nucleotide polymorphism were investigated by polymerase chain reaction and direct sequencing in a Chinese cohort that included 464 infertile men with idiopathic azoospermia or oligospermia and 458 controls with normal fertility. Overall, no significant differences in the distributions of the genotypes of the MTHFR rs55763075 polymorphism were detected between the infertility and control groups. A statistically significant increased risk of male infertility was found for carriers
\end{abstract}


of the rs55763075 AA genotype when compared with homozygous carriers of the rs55763075 GG genotype in the azoospermia subgroup $(\mathrm{OR}=1.721 ; 95 \% \mathrm{CI}=1.055-2.807 ; \mathrm{P}=0.031)$. Furthermore, we found that rs55763075 was associated with folate and homocysteine levels in patients with idiopathic azoospermia. Our results indicated that the MTHFR 3'-UTR rs55763075 polymorphism might modify the susceptibility to male infertility with idiopathic azoospermia.

Key words: MiR-34b; 3'-UTR; Methylenetetrahydrofolate reductase; Male infertility

\section{INTRODUCTION}

Infertility is defined as the failure to achieve a clinically recognized pregnancy despite having 12 months or more of regular unprotected sex (Huang et al., 2012). Infertility is a worldwide reproductive health problem and affects approximately $10-15 \%$ of couples. It is estimated that approximately $50 \%$ of couple infertility is due to male infertility (De Kretser and Baker, 1999). Although several causes have been identified for impaired male fertility, the etiology of male infertility remains poorly understood. There is a growing body of evidence that genetic abnormalities might account for $15-30 \%$ of male factor infertility through affecting the function or expression of proteins involved in male reproduction (O'Flynn O'Brien et al., 2010). Mutation in key testis regulatory genes, for example, in combination with environmental factors, might be responsible for poor sperm quality and reduced sperm numbers (Eloualid et al., 2012). Therefore, it is important to identify genetic polymorphisms and risk factors that are associated with male infertility.

Several genes are known to play roles in orchestrating the complex process of spermatogenesis (Visser and Repping, 2010). Furthermore, folate plays a key role in cellular physiology by participating in DNA synthesis, repair, and methylation, and in the maintenance and stability of the genome. Human methylenetetrahydrofolate reductase (MTHFR) is an integral enzyme in the folate pathway that catalyzes the reduction of 5,10-methylenetetrahydrofolate to 5-methyltetra-hydrofolate (Goyette et al., 1994). Recent research has further emphasized the role of the MTHFR gene in male fertility. A study on adult mice demonstrated that MTHFR plays a critical role in spermatogenesis (Chen et al., 2001), and it has also been demonstrated that MTHFR deficient mice exhibit hyperhomocysteinemia and compromised spermatogenesis (Kelly et al., 2005). There is increasing evidence that common variants of MTHFR are associated with lower enzyme activity, lower red blood cell and plasma folate, and elevated plasma homocysteine (Shen et al., 2001). Several studies have found that polymorphisms in the MTHFR gene were associated with altered MTHFR enzyme activity and hyperhomocysteinemia, which was considered as a risk factor for different disorders including infertility (Austin et al., 2004). However, studies investigating the role of MTHFR polymorphisms in patients with male infertility have yielded conflicting results. Some studies have suggested that MTHFR polymorphisms (C677T, A1298C, and G1793A) are risks for human male infertility (Gava et al., 2011; Safarinejad et al., 2011), whereas others have not supported such an association (Ravel et al., 2009; Montjean et al., 2011).

MicroRNAs (miRNAs) are a class of endogenous noncoding RNAs, 18-25 nt in length, 
that can regulate the expression of target mRNAs through base pairing to target sequences in their 3'-UTRs (Bartel, 2004). It is considered reasonable to hypothesize, therefore, that single nucleotide polymorphisms (SNPs) at miRNA-binding sites might alter the strength of miRNA binding, impacting the expression of miRNA targets and hence influencing the development of numerous complex diseases (Sethupathy and Collins, 2008). In addition, SNPs in the premiRNA or mature miRNA sequences themselves could modulate miRNA-target interactions through alteration of miRNA expression or maturation (Wang et al., 2008; Ryan et al., 2010). Wu et al. (2013) found that a miR-149 binding site SNP in the 3'-UTR of MTHFR increased coronary heart disease risk through modification of miRNA binding. In a pilot study, we identified a SNP (rs55763075) in a miR-34b binding site in MTHFR using a bioinformatic approach. Increasing evidence has suggested that miR-34b is involved in various biological processes such as spermatogenesis (Abu-Halima et al., 2013a,b). We hypothesized, therefore, that polymorphic variation in the miR-34b binding site might alter the strength of miR-34b binding to regulate the expression of MTHFR and hence influence the process of spermatogenesis.

There are, however, currently no studies that have investigated the association between miR-34b-binding site polymorphisms and idiopathic male infertility. In this study, we test the hypothesis that SNP in miR-34b-binding sites of MTHFR might interfere with the activity of MTHFR and modulate the risk of idiopathic male infertility in a Chinese population.

\section{MATERIAL AND METHODS}

\section{Subjects and sample collection}

This study was approved by the Ethics Committee of Zhejiang Medical University. We recruited 464 patients with infertility including 253 men with idiopathic azoospermia and 211 with oligospermia (sperm count less than $15 \times 10^{6} / \mathrm{mL}$ ) from the Andrology Outpatient Clinic of the Centre of Clinical Reproductive Medicine between March 2011 and April 2013, for a case-control study. Patients having diseases known to affect spermatogenesis, such as maldescensus of the testis, orchitis, or obstruction of the vas deferens and varicocele were excluded from this study. The control group consisted of 458 fertile men who were fathers of at least one child and who reported no history of assisted reproductive technologies. Blood donated from each subject $(5 \mathrm{~mL})$ was used to extract genomic DNA; a routine semen analysis was also performed. The analysis of semen was performed according to World Health Organization guidelines (Lu et al., 2010).

\section{Genotyping}

Genomic DNA was extracted from peripheral blood mononuclear cells using the QiaAmp DNA Blood Mini kit (Qiagen, Valencia, CA, USA). The genotypes of rs55763075 were determined by direct PCR sequencing. All PCR reagents were purchased from TaKaRa Biotechnology Co., Ltd. (Dalian, China). Primer sequences used for amplifications were as follows: Forward: 5'-TGA TGC CCT TGC GTG TTT TG-3'; reverse: 5'-GCC AGG AGT CTG TGC TCT TT-3'. Reaction conditions were as follows: initial denaturation at $94^{\circ} \mathrm{C}$ for $3 \mathrm{~min}$; 35 cycles of denaturation at $94^{\circ} \mathrm{C}$ for $30 \mathrm{~s}$, primer annealing at $58^{\circ} \mathrm{C}$ for $30 \mathrm{~s}$, extension at $72^{\circ} \mathrm{C}$ for $1 \mathrm{~min}$; followed by a final extension step of $72^{\circ} \mathrm{C}$ for $10 \mathrm{~min}$. PCR products were puri- 
fied using the QIAQuick PCR purification kit and examined using the automatic sequencer ABI3730XL (Applied Biosystems).

\section{Detection of folate and homocysteine (Hcy) levels}

Serum folate levels were determined by enzyme-linked immunosorbent assay (ELISA) (Boster Biotech., Wuhan, China) using an iMark ${ }^{\mathrm{TM}}$ microplate absorbance reader (BioRad, Hercules, CA, USA). Plasma Hcy levels were determined by chemiluminescence using an Abbott Laboratories IMX Blood Chemistry Analyzer (Abbott Laboratories, Irving, Texas, USA).

\section{Statistical analyses}

Statistical analysis was carried out using the statistical software SPSS version 12.0 (SPSS Inc., Chicago, IL, USA). Infertility risks were estimated as odds ratios (ORs) and their respective $95 \%$ confidence intervals (CIs) using unconditional multivariate logistic regression. All P values were two-sided; a P value $<0.05$ was considered to be statistically significant.

\section{RESULTS}

\section{Characteristics of the study population}

The baseline demographics, plasma Hcy levels, and serum folate levels of the study subjects are shown in Table 1. There were no significant differences in age or body mass index between the men with infertility and the normal controls. Serum folate levels were significantly decreased in the men with infertility compared to the controls; in contrast, the men with infertility exhibited increased plasma Hcy levels as compared to the subjects with normal fertility.

Table 1. Characteristics of the study population.

\begin{tabular}{lcr}
\hline & Infertile men & Normal fertile donors \\
\hline Number & 464 & 458 \\
Age (years) & $36.4 \pm 7.2$ & $35.8 \pm 6.9$ \\
BMI $\left(\mathrm{kg} / \mathrm{m}^{2}\right)$ & $27.4 \pm 4.5$ & $26.8 \pm 5.1$ \\
Folate levels $(\mathrm{ng} / \mathrm{mL})$ & $7.7 \pm 2.8$ & $11.3 \pm 3.7$ \\
Hcy levels & $14.2 \pm 4.2$ & $8.5 \pm 2.1$ \\
\hline
\end{tabular}

Data $=$ means $\pm \mathrm{SD} ; \mathrm{BMI}=$ body mass index; Hcy $=$ homocysteine.

\section{Association between the MTHFR rs55763075 polymorphism and male infertility}

The frequencies of the MTHFR 3'-UTR rs55763075 genotypes in patients and controls are shown in Table 2. All the rs55763075 genotype distributions (normal fertile controls: $\chi^{2}=2.204, \mathrm{P}=0.138$; infertile participants: $\chi^{2}=0.296, \mathrm{P}=0.586$ ) were in agreement with Hardy-Weinberg equilibrium. Overall, no significant differences in the distributions of the MTHFR rs55763075 genotypes were observed between the men with infertility and the control group. The infertility group was further stratified into two subgroups: subjects with idio- 
pathic azoospermia or with oligozoospermia (Table 3). Compared to the control group, a statistically significant increased risk of male infertility was found in the azoospermia subgroup for carriers of the rs55763075 AA genotype of MTHFR when compared with homozygous carriers of rs $55763075 \mathrm{GG}$ genotype $(\mathrm{OR}=1.721 ; 95 \% \mathrm{CI}=1.055-2.807 ; \mathrm{P}=0.031)$, while in the oligozoospermia group, the genotype frequency distribution showed no significant difference. These results suggested that the AA genotype of rs55763075 (G>A) might contribute to idiopathic azoospermia.

Table 2. Association of rs55763075 with the risk of male infertility.
\begin{tabular}{lcccc}
\hline Polymorphism & Infertile men $(\mathrm{N}=464)$ & Normal fertile donors $(\mathrm{N}=458)$ & OR $(95 \% \mathrm{CI})$ & P value \\
\hline GG & $176(37.9 \%)$ & $192(41.9 \%)$ & 1.0 (Reference) & \\
GA & $224(48.3 \%)$ & $220(48.0 \%)$ & $1.111(0.842-1.465)$ & 0.481 \\
AA & $64(13.8 \%)$ & $46(10.0 \%)$ & $1.518(0.987-2.334)$ & 0.065 \\
G allele & $576(62.1 \%)$ & $604(65.9 \%)$ & 1.0 (Reference) & \\
A allele & $352(37.9 \%)$ & $312(34.1 \%)$ & $1.183(0.978-1.431)$ & 0.090 \\
\hline
\end{tabular}

$\mathrm{OR}=$ odds ratio; $\mathrm{CI}=$ confidence interval.

Table 3. Subgroup analysis of the MTHFR gene polymorphism and the risk of male infertility.

\begin{tabular}{|c|c|c|c|c|c|}
\hline Infertility class & Polymorphism & Infertile men & Normal fertile donors $(\mathrm{N}=458)$ & OR $(95 \% \mathrm{CI})$ & $P$ value \\
\hline \multirow[t]{5}{*}{ Oligozoospermia } & GG & $81 \quad(38.4 \%)$ & $192(41.9 \%)$ & 1.0 (Reference) & \\
\hline & GA & $108(51.2 \%)$ & $220(48.0 \%)$ & $1.193(0.842-1.691)$ & 0.331 \\
\hline & $\mathrm{AA}$ & $22(10.4 \%)$ & $46 \quad(10.0 \%)$ & $1.268(0.725-2.217)$ & 0.465 \\
\hline & G allele & $266(63.0 \%)$ & $604(65.9 \%)$ & 1.0 (Reference) & \\
\hline & A allele & $156(37.0 \%)$ & $312(34.1 \%)$ & $1.183(0.978-1.431)$ & 0.090 \\
\hline \multirow[t]{5}{*}{ Idiopathic azoospermia } & GG & $95 \quad(37.6 \%)$ & $192(41.9 \%)$ & 1.0 (Reference) & \\
\hline & GA & $116(45.8 \%)$ & $220(48.0 \%)$ & $1.044(0.749-1.455)$ & 0.866 \\
\hline & $\mathbf{A A}$ & $42(16.6 \%)$ & $46(10.0 \%)$ & $1.721(1.055-2.807)$ & 0.031 \\
\hline & $\mathrm{G}$ allele & $306(60.5 \%)$ & $604(65.9 \%)$ & 1.0 (Reference) & \\
\hline & A allele & $200(39.5 \%)$ & $312(34.1 \%)$ & $1.265(1.011-1.584)$ & 0.043 \\
\hline
\end{tabular}

$\overline{\mathrm{OR}}=$ odds ratio; $\mathrm{CI}=$ confidence interval; bold text indicates there exist significance between infertile man and normal donors $(\mathrm{P}<0.05)$.

\section{Association between the MTHFR rs55763075 polymorphism and serum folate and Hcy levels}

Rs55763075 might modify the binding of human miR-34b to MTHFR the as the variant creates a miR-34b binding site in the MTHFR 3'-UTR (Figure 1A). Therefore, we detected the serum folate and Hcy levels in patients with azoospermia with different rs55763075 genotypes. As shown in Figure 1B and C, we observed that the presence of the rs55763075 AA genotype was associated with decreased folate levels and increased Hcy levels in patients with azoospermia. The results indicated that the SNP rs55763075 might influence MTHFR function.

\section{DISCUSSION}

Although genetic factors play a role in the etiology of idiopathic male infertility, the biochemical abnormalities underlying the predisposition to infertility and the exact pathophysiology of impaired spermatogenesis remain to be elucidated. Several studies have shown 
that individual susceptibility to infertility is likely due to MTHFR polymorphism (Mfady et al., 2014). In current study, we found that a single nucleotide polymorphism (rs55763075) in a miR-34b binding site in the MTHFR 3'-UTR was associated with idiopathic azoospermia in a Han Chinese population.

A

$$
\operatorname{miR}-34 b
$$

MTHFR 3' UTR 288-306

miRNA: $3^{\prime}$ uaCCGUCACCUCAAUCACUAAc 5'

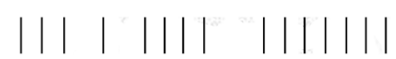

untranslated region: 5' aaGGC -GAGGAG - -AGUGAUUg 3'

rs55763075 $\rightarrow \mathrm{A}$

B

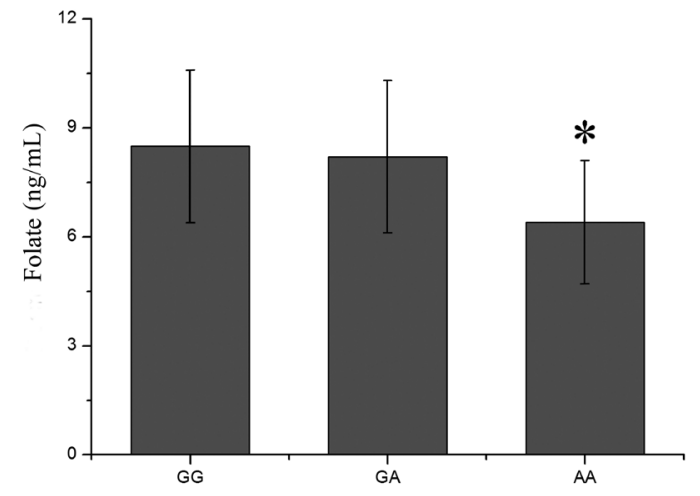

C

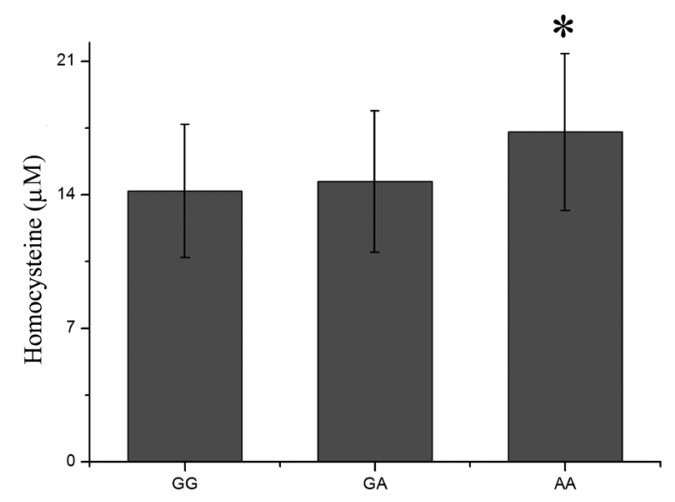

Figure 1. Association between the MTHFR rs55763075 polymorphism and folate and Hcy levels. A. miRNASNP 2.0 (http://www.bioguo.org/miRNASNP/) predicted that rs55763075 could create a miR-34b-mRNA binding site in the MTHFR 3'-UTR. B. Serum vitamin folate levels in patients with azoospermia. C. Plasma Hcy levels in patients with azoospermia. Open triangle $=$ the site of polymorphism; $* \mathrm{P}<0.05$. 
DNA methylation is the primary epigenetic phenomenon regulating gene expression and genome integrity and is critical for the correct development of sperm. MTHFR is one of the key regulatory enzymes of DNA synthesis and methylation metabolism. There is an increasing number of published reports on the association between MTHFR polymorphisms and the risk of unexplained male infertility. C677T, the most commonly studied MTHFR SNP, has been demonstrated to be associated with male infertility in certain populations but not in others (Bezold et al., 2001; Stuppia et al., 2003; A et al., 2007; Chellat et al., 2012; Vani et al., 2012). Several meta-analyses have suggested that C677T increases the risk of infertility (Tuttelmann et al., 2007; Gupta et al., 2011); however, a recent meta-analysis from Wu et al. (2012) suggested that this SNP was only significantly associated with a susceptibility to male infertility in Asians, but not in Caucasians. Another study involving infertile and fertile and/or normospermic individuals of Korean ancestry found that the MTHFR C677T, Methionine Synthase Reductase (MTRR) A66G and methionine synthase (MS) A2756G genotypes were independently associated with male infertility (Lee et al., 2006). Conversely, a study of 77 subfertile men of Dutch ancestry found the C allele of the C677T polymorphism was not associated with male factor infertility (Ebisch et al., 2003). One explanation for these conflicting results from studies on different geographic populations suggested that the role of the C677T variant in the susceptibility to male infertility might depend on other factors such as total folate intake level, DNA collection and genotyping method, sample size, and ethnic group (Ebisch et al., 2007). In the present study, we found that a genetic variation in the miR$34 \mathrm{~b}$ binding site in the 3'-UTR of MTHFR (rs55763075) was a risk factor for male infertility with idiopathic azoospermia. Therefore, our results indicted that MTHFR polymorphism was associated with spermatogenic impairment.

MiRNAs are a family of small noncoding RNAs of approximately 22 nucleotides that are recognized as endogenous physiological regulators of gene expression via base pair binding to the 3'-UTR of their target mRNAs (Stark et al., 2008). There is increasing evidence that suggests that miRNAs might also play important roles in mammalian spermatogenesis, and in fact a series of miRNAs are expressed abundantly in male germ cells throughout spermatogenesis (He et al., 2009; Yan et al., 2009). The term miR-SNP refers to SNPs in the miRNA coding sequence, whereas miR-TS-SNP is defined as a SNP occurring in the miRNA target site (TS) or binding site. Many studies have revealed that genetic variances in microRNA genes and their TSs are associated with complex genetic diseases (Sethupathy et al., 2007; Saetrom et al., 2009). However, the association between miR-SNPs or miR-TS-SNPs with male fertility remains unclear. In a study from Zhang et al. (2011), SNPs in the miRNA-binding sites of genes for male infertility were analyzed to explore the possible association between SNPs occurring at miRNA-binding sites and idiopathic male infertility. Their results indicated that SNPs residing in miRNA-binding sites of Chorionic Gonadotropin Alpha Polypeptide (CGA) increased the risk of idiopathic male infertility. Recently, several studies have found that miRNA-34b in particular was associated with male infertility. A study from Abu-Halima et al. (2013b) demonstrated that miR-34b, miR-122, and miR-1973 exhibited the highest fold changes in samples from asthenozoospermic men. In the present study, using the online bioinformatic analysis tool TargetScan, we identified a single nucleotide polymorphism (rs55763075) in a miR-34b binding site in the MTHFR gene 3'-UTR and assessed whether there was any association between rs55763075 genotype and male fertility status. Our results demonstrated a statistically significant increased risk of male infertility in the azoospermia subgroup for car- 
riers of the MTHFR rs55763075 AA genotype when compared with homozygous carriers of the rs55763075GG genotype. Furthermore, the rs55763075 AA genotype was associated with decreased folate levels and increased Hcy levels in patients with azoospermia as well. Our findings indicated that the SNP rs55763075 might influence MTHFR function.

In conclusion, in the present study, we found that genetic variation in a miR-34b binding site in the 3'-UTR of MTHFR is a risk factor for azoospermia. The validation of our results in larger patient groups might provide evidence for the contribution of rs55763075 genotypes to the efficiency of spermatogenesis.

\section{ACKNOWLEDGMENTS}

The authors gratefully acknowledge the members of the Reproductive Medicine Center, First Affiliated Hospital of Zhejiang University for collecting the semen analysis data. Research supported by grants for Scientific Research Projects of Zhejiang Provincial Department of Education (Grant \#N20130393) and the Social Development Project of Zhenjiang (Grant \#SH 2014093).

\section{REFERENCES}

A ZC, Yang Y, Zhang SZ, Li N, et al. (2007). Single nucleotide polymorphism C677T in the methylenetetrahydrofolate reductase gene might be a genetic risk factor for infertility for Chinese men with azoospermia or severe oligozoospermia. Asian J. Androl. 9: 57-62.

Abu-Halima M, Backes C, Leidinger P, Keller A, et al. (2013a). MicroRNA expression profiles in human testicular tissues of infertile men with different histopathologic patterns. Fertil. Steril. 101: 78-86e2.

Abu-Halima M, Hammadeh M, Schmitt J, Leidinger P, et al. (2013b). Altered microRNA expression profiles of human spermatozoa in patients with different spermatogenic impairments. Fertil. Steril. 99: 1249-1255e16.

Austin RC, Lentz SR and Werstuck GH (2004). Role of hyperhomocysteinemia in endothelial dysfunction and atherothrombotic disease. Cell Death Differ. 11: S56-S64.

Bartel DP (2004). MicroRNAs: genomics, biogenesis, mechanism, and function. Cell 116: 281-297.

Bezold G, Lange M and Peter RU (2001). Homozygous methylenetetrahydrofolate reductase C677T mutation and male infertility. N. Engl. J. Med. 344: 1172-1173.

Chellat D, Rezgoune ML, Hamane D, Semmame O, et al. (2012). Influence of methylenetetrahydrofolate reductase C677T gene polymorphisms in Algerian infertile men with azoospermia or severe oligozoospermia. Genet. Test. Mol. Biomarkers 16: 874-878.

Chen Z, Karaplis AC, Ackerman SL, Pogribny IP, et al. (2001). Mice deficient in methylenetetrahydrofolate reductase exhibit hyperhomocysteinemia and decreased methylation capacity, with neuropathology and aortic lipid deposition. Hum. Mol. Genet. 10: 433-443.

De Kretser DM and Baker HW (1999). Infertility in men: recent advances and continuing controversies. J. Clin. Endocrinol. Metab. 84: 3443-3450.

Ebisch IM, van Heerde WL, Thomas CM, van der Put N, et al. (2003). C677T methylenetetrahydrofolate reductase polymorphism interferes with the effects of folic acid and zinc sulfate on sperm concentration. Fertil. Steril. 80 : 1190-1194.

Ebisch IM, Thomas CM, Peters WH, Braat DD, et al. (2007). The importance of folate, zinc and antioxidants in the pathogenesis and prevention of subfertility. Hum. Reprod. Update 13: 163-174.

Eloualid A, Abidi O, Charif M, El Houate B, et al. (2012). Association of the MTHFR A1298C variant with unexplained severe male infertility. PLoS One 7: e34111.

Gava MM, Chagas Ede O, Bianco B, Christofolini DM, et al. (2011). Methylenetetrahydrofolate reductase polymorphisms are related to male infertility in Brazilian men. Genet. Test. Mol. Biomarkers 15: 153-157.

Goyette P, Sumner JS, Milos R, Duncan AM, et al. (1994). Human methylenetetrahydrofolate reductase: isolation of cDNA mapping and mutation identification. Nat. Genet. 7: 551.

Gupta N, Gupta S, Dama M, David A, et al. (2011). Strong association of 677 C $>$ T substitution in the MTHFR gene with male infertility - a study on an Indian population and a meta-analysis. PLoS One 6: e22277. 
He Z, Kokkinaki M, Pant D, Gallicano GI, et al. (2009). Small RNA molecules in the regulation of spermatogenesis. Reproduction 137: 901-911.

Huang C, Liu W, Ji GX, Gu AH, et al. (2012). Genetic variants in TP53 and MDM2 associated with male infertility in Chinese population. Asian J. Androl. 14: 691-694.

Kelly TL, Neaga OR, Schwahn BC, Rozen R, et al. (2005). Infertility in 5,10-methylenetetrahydrofolate reductase (MTHFR)-deficient male mice is partially alleviated by lifetime dietary betaine supplementation. Biol. Reprod. 72: 667-677.

Lee HC, Jeong YM, Lee SH, Cha KY, et al. (2006). Association study of four polymorphisms in three folate-related enzyme genes with non-obstructive male infertility. Hum. Reprod. 21: 3162-3170.

Lu JC, Huang YF and Lü NQ (2010). WHO Laboratory Manual for the Examination and Processing of Human Semen: its applicability to andrology laboratories in China. Zhonghua Nan Ke Xue 16: 867-871.

Mfady DS, Sadiq MF, Khabour OF, Fararjeh AS, et al. (2014). Associations of variants in MTHFR and MTRR genes with male infertility in the Jordanian population. Gene 536: 40-44.

Montjean D, Benkhalifa M, Dessolle L, Cohen-Bacrie P, et al. (2011). Polymorphisms in MTHFR and MTRR genes associated with blood plasma homocysteine concentration and sperm counts. Fertil. Steril. 95: 635-640.

O'Flynn O'Brien KL, Varghese AC and Agarwal A (2010). The genetic causes of male factor infertility: a review. Fertil. Steril. 93: 1-12.

Ravel C, Chantot-Bastaraud S, Chalmey C, Barreiro L, et al. (2009). Lack of association between genetic polymorphisms in enzymes associated with folate metabolism and unexplained reduced sperm counts. PLoS One 4: e6540.

Ryan BM, Robles AI and Harris CC (2010). Genetic variation in microRNA networks: the implications for cancer research. Nat. Rev. Cancer 10: 389-402.

Saetrom P, Biesinger J, Li SM, Smith D, et al. (2009). A risk variant in an miR-125b binding site in BMPR1B is associated with breast cancer pathogenesis. Cancer Res. 69: 7459-7465.

Safarinejad MR, Shafiei N and Safarinejad S (2011). Relationship between genetic polymorphisms of methylenetetrahydrofolate reductase (C677T, A1298C, and G1793A) as risk factors for idiopathic male infertility. Reprod. Sci. 18: 304-315.

Sethupathy P and Collins FS (2008). MicroRNA target site polymorphisms and human disease. Trends Genet. 24: 489497.

Sethupathy P, Borel C, Gagnebin M, Grant GR, et al. (2007). Human microRNA-155 on chromosome 21 differentially interacts with its polymorphic target in the AGTR1 3'-untranslated region: a mechanism for functional singlenucleotide polymorphisms related to phenotypes. Am. J. Hum. Genet. 81: 405-413.

Shen H, Spitz MR, Wang LE, Hong WK, et al. (2001). Polymorphisms of methylene-tetrahydrofolate reductase and risk of lung cancer: a case-control study. Cancer Epidemiol. Biomarkers Prev. 10: 397-401.

Stark A, Bushati N, Jan CH, Kheradpour P, et al. (2008). A single Hox locus in Drosophila produces functional microRNAs from opposite DNA strands. Genes Dev. 22: 8-13.

Stuppia L, Gatta V, Scarciolla O, Colosimo A, et al. (2003). The methylenetethrahydrofolate reductase (MTHFR) C677T polymorphism and male infertility in Italy. J. Endocrinol. Invest. 26: 620-622.

Tuttelmann F, Rajpert-De Meyts E, Nieschlag E and Simoni M (2007). Gene polymorphisms and male infertility - a metaanalysis and literature review. Reprod. Biomed. Online 15: 643-658.

Vani GT, Mukesh N, Rama Devi P, Usha Rani P, et al. (2012). Methylenetetrahydrofolate reductase C677T polymorphism is not associated with male infertility in a South Indian population. Andrologia 44: 252-259.

Visser L and Repping S (2010). Unravelling the genetics of spermatogenic failure. Reproduction 139: 303-307.

Wang G, van der Walt JM, Mayhew G, Li YJ, et al. (2008). Variation in the miRNA-433 binding site of FGF20 confers risk for Parkinson disease by overexpression of alpha-synuclein. Am. J. Hum. Genet. 82: 283-289.

Wu C, Gong Y, Sun A, Zhang Y, et al. (2013). The human MTHFR rs4846049 polymorphism increases coronary heart disease risk through modifying miRNA binding. Nutr. Metab. Cardiovasc. Dis. 23: 693-698.

Wu W, Shen O, Qin Y, Lu J, et al. (2012). Methylenetetrahydrofolate reductase C677T polymorphism and the risk of male infertility: a meta-analysis. Int. J. Androl. 35: 18-24.

Yan N, Lu Y, Sun H, Qiu W, et al. (2009). Microarray profiling of microRNAs expressed in testis tissues of developing primates. J. Assist. Reprod. Genet. 26: 179-186.

Zhang H, Liu Y, Su D, Yang Y, et al. (2011). A single nucleotide polymorphism in a miR-1302 binding site in CGA increases the risk of idiopathic male infertility. Fertil. Steril. 96: 34-39. 